\title{
Actinomicosis pulmonar, una enfermedad escasa
}

\section{Pulmonary actinomycosis, a rare disease}

Leidy Daniela Guarín-Acevedo ${ }^{1}$, Lizeth LaRa-BáeZ ${ }^{1}$, Yardany Rafael MÉndez-Fandiño².

\section{Resumen}

INTRODUCCIÓN: la actinomicosis es una enfermedad crónica poco común, causada principalmente por actinomicetos anaerobios grampositivos conocidos como Actinomyces israelii. Normalmente habitan en la orofaringe, el tracto gastrointestinal y el genitourinario, por lo cual pueden llegar a aislarse en el $12-52 \%$ de los individuos sanos. En este caso, se presenta un paciente con actinomicosis pulmonar.

DESCRIPCIÓN DEL CASO: se trata de un paciente masculino de 81 años con antecedentes de masa pulmonar, enfermedad pulmonar obstructiva crónica (EPOC) y tabaquismo; el paciente es reconsultante por cuadros clínicos consistente con alzas térmicas no cuantificadas, tos con expectoración verde y movilización de secreciones, y disnea de medianos esfuerzos. Durante las hospitalizaciones se le realizaron estudios de tomografía por emisión de positrones/tomografía computarizada (PET/CT) y una biopsia guiada por tomografía, que evidenciaron lesiones hipermetabólicas y colonias de Actinomyces con granulomas no necrotizantes, respectivamente, hallazgos compatibles con el cuadro clínico descrito. Por esta razón, se le diagnostica neumonía crónica granulomatosa no necrotizante, con numerosas colonias de Actinomyces, y se inicia el tratamiento con amoxicilina durante 6 meses.

DISCUSIÓN: a menudo, la sintomatología de un paciente con actinomicosis se confunde con una neoplasia o tuberculosis, debido a que el diagnóstico es difícil de establecer, ya que inicialmente presenta sintomatología e imágenes inespecíficas. El diagnóstico se establece en muchas ocasiones después de haber agotado un importante número de pruebas complementarias, incluyendo la biopsia pulmonar.

Palabras clave: actinomicosis pulmonar, infección por Actinomyces, Actinomyces israelii, masa pulmonar, gránulos de azufre.

\section{Abstract}

INTRODUCTION: actinomycosis is a rare chronic disease, caused mainly by grampositive anaerobic actinomycetes known as Actinomyces israelii. It normally inhabits the oropharynx, the gastrointestinal and genitourinary tracts, and can therefore be isolated in 12 to $52 \%$ of healthy individuals. In this case, a patient with pulmonary actinomycosis is presented.

CASE DESCRIPTION: this is an 81-year-old male patient with a history of lung mass, Chronic Obstructive Pulmonary Disease (COPD), and smoking; the patient is reconsultant due to clinical pictures consisting of unquantified thermal rises, cough with green expectoration and mobilization of secretions, dyspnea of medium efforts. During hospitalizations,
${ }^{1}$ Facultad de Medicina, Universidad Pedagógica y Tecnológica de Colombia. Tunja, Colombia.

${ }^{2}$ Medicina Interna, Metodología de la Investigación, Universidad Pedagógica y Tecnológica de Colombia. Duitama, Colombia.

Autor de correspondencia Yardany Rafael Méndez-Fandiño.

Correo electrónico:

yardanymendezf@yahoo.es

Recibido: 03/08/2020

Aceptado:25/08/2020 
Positron Emission Tomography/Computed Tomography (PET/CT) studies and tomography-guided biopsy were performed, which showed hypermetabolic lesions and actinomyces colonies with non-necrotizing granulomas, respectively, findings compatible with the clinical picture described, making a diagnosis of chronic non-necrotizing granulomatous pneumonia. with numerous actinomyces colonies and treatment with amoxicillin is started for 6 months.

DISCUSSION: often the symptomatology of a patient with actinomycosis is confused with a neoplasm or tuberculosis because the diagnosis is difficult to establish, since symptoms and nonspecific images are initially presented. The diagnosis is established on many occasions after having exhausted a significant number of complementary tests, including a lung biopsy.

Keywords: Pulmonary Actinomycosis; Actinomyces Infection; Actinomyces israelii; Lung Mass; Sulfur Granules.

\section{Introducción}

La actinomicosis pulmonar es una enfermedad extremadamente rara de característica granulomatosa, crónica, de progresión lenta, ocasionada por bacterias filamentosas anaeróbicas grampositivas de la familia Actinomycetaceae, pertenecientes al género Actinomyces (1).

Normalmente, Actinomyces sp. habita en la orofaringe, el tracto gastrointestinal y el tracto genitourinario, y se aísla en el 12-52 \% de los individuos sanos; por esta razón, suele afectar las regiones cervicofaciales, torácicas, abdominales y perineales, donde se pueda presentar un estancamiento ya sea de materia fecal o esputo (2).

El Actinomyces israelii se vuelve patógeno cuando penetra en los tejidos blandos; aunque la mayoría de estas infecciones son polimicrobianas, una vez se produce la ruptura de la barrera mucosa puede originar una infección en casi cualquier parte del organismo con un único patógeno, lo que da lugar a los tres tipos de presentación en los que se clasifica esta enfermedad: cervicofacial, abdominopélvico y torácico. La forma torácica constituye el 15-45\% del total de las formas de presentación, y su causa más frecuente es la aspiración de los microorganismos de la orofaringe, que adopta formas de tipo endobronquial o pleural. En la actualidad ambas presentaciones son infrecuentes; tanto en la literatura nacional como en la internacional se ha publicado un número muy limitado de casos (3-6).

Epidemiológicamente, las infecciones por Actinomyces no son tan usuales y se consideran raras. La incidencia de todas las formas de actinomicosis ha descendido en las últimas 3-4 décadas, probablemente por el uso temprano de antibióticos ante la sospecha de una infección y una mejor higiene dental de la población, con un número muy reducido de casos publicados. Cabe resaltar que no existe predilección estacional, racial u ocupacional. Entre 2002-2003, el Departamento de Salud del Reino Unido informó que el 0,0006 \% de las consultas en los hospitales ingleses fueron por actinomicosis. En cuanto a la epidemiología, en Colombia, debido a la falta de datos para la estimación de la incidencia de actinomicosis, esta se considera mínima, por lo que el número reducido de casos publicados sobre esta patología motiva a la publicación académica $(1,7,8)$.

\section{Presentación del caso}

Es un paciente masculino de 81 años, usuario de oxígeno domiciliario, quien asiste al servicio de urgencias por un cuadro clínico de 8 días de evolución consistente con alzas térmicas no cuantificadas asociadas con tos con expectoración verde y movilización de secreciones. El paciente presenta una masa localizada en el pulmón, la cual se encuentra en estudio desde hace un mes; además, presenta otros antecedentes relevantes como enfermedad pulmonar obstructiva crónica (EPOC), consumo de 10 paquetes de cigarrillo por año durante 10 años, cuyo último consumo fue hace 20 años, y consumo de alcohol en entornos sociales.

En la revisión por sistemas, el paciente refiere disnea a medianos esfuerzos. Los signos vitales de ingreso son: presión arterial (PA): 110/60 mm Hg, frecuencia cardíaca (FC): 113 lpm, frecuencia respiratoria (FR): 
$20 \mathrm{rpm}$, temperatura $\left(\mathrm{T}^{\mathrm{o}}\right): 36^{\circ} \mathrm{C}$. Al examen físico se evidencia un uso de la musculatura accesoria en la respiración dada por la presencia de tirajes intercostales, respiración jadeante y ruidos respiratorios con roncus, sibilancias e hipoventilación en la base derecha.

El paciente aporta paraclínicos extrainstitucionales que reportan: nódulos pulmonares finos diseminados en ambos campos pulmonares, de predominio peribroncovascular, evidenciados en una tomografía axial computarizada (TAC) de tórax simple del año anterior; una masa de contornos bien definidos con un área de densidad heterogénea adyacente a la aorta ascendente del segmento anterior del lóbulo superior derecho, de $47 \mathrm{~mm}$, que se extiende hasta el hilio pulmonar derecho; además, una biopsia de la mucosa bronquial del lóbulo superior derecho tomada tres años antes, en la cual se encuentran fragmentos de mucosa bronquial con una grave inflamación crónica granulomatosa epitelioides sin necrosis de caseificación, cambios epiteliales reactivos asociados y material fibrinoide con neutrófilos adyacentes. En el material examinado la muestra es negativa para malignidad, y las tinciones de PSA y Ziehl-Neelsen (ZN) también son negativas. Por esta razón, se considera que los hallazgos sugieren la presencia de cáncer de pulmón frente a enfermedad granulomatosa, lo cual está por definir.

Institucionalmente, se solicita una prueba de los niveles de sodio, potasio, nitrógeno ureico en sangre (BUN) y creatinina, cuyos resultados son normales; un cuadro hemático, que muestra leucocitosis en 11,690 a expensas de neutrófilos con el $76 \%$; y una radiografía de tórax (Figura 1A).

El TAC extrainstitucional que evidenció la masa más el antecedente de EPOC y la radiografía de tórax institucional se consideran compatibles con una bronquitis. Inicialmente, se piensa en una EPOC dependiente de oxígeno sobreinfectada exacerbada (bronquiolitis bacteriana aguda), por lo que se inicia el tratamiento farmacológico con beclometasona dipropionato de 250 mcg, acetaminofén en tabletas de $500 \mathrm{mg}$, bromuro de ipratropio en aerosol de $0,02 \mathrm{mg} /$ dosis, hidrocortisona (succinato sódico) en polvo para reconstituir de 100 $\mathrm{mg}$, ampicilina $1 \mathrm{~g} /$ sulbactam $0,5 \mathrm{~g}$ sódica en polvo para reconstituir administrado por vía intravenosa, doxiciclina en tableta de $100 \mathrm{mg}$, heparina de $40 \mathrm{mg}$, omeprazol en cápsula de $20 \mathrm{mg}$ y oxígeno gaseoso a $1 \mathrm{~L} / \mathrm{min}$. Se emite una orden de hospitalización al paciente $\mathrm{y}$, por trámites administrativos, se realiza una remisión a otra institución para continuar con los estudios y el tratamiento de sus patologías. Cuatro meses después, el paciente se remite al Instituto Nacional de Cancerología por el hallazgo incidental de la masa pulmonar encontrada un año antes.

Al paciente se le realiza un estudio PET/CT que muestra unas lesiones hipermetabólicas; por tanto, se le realiza una biopsia guiada por tomografía, que mostró colonias de Actinomyces con cambios histopatológicos compatibles, parénquima pulmonar con compromiso inflamatorio crónico en forma de granulomas no necrotizantes, infiltrado intersticial de linfocitos, plasmocitos y gránulos de azufre. Las estructuras encontradas son positivas para Gomori y PAS, pero negativas para $\mathrm{ZN}$. Además, se tomó una radiografía de tórax (Figura 1B). Con base en lo anterior, se diagnostica neumonía crónica granulomatosa no necrotizante, con numerosas colonias de actinomices, y se inicia el tratamiento con amoxicilina de $500 \mathrm{mg}$ cada 8 horas durante 6 meses.

Nuevamente, cuatro meses después, el paciente ingresa a la institución para el control de los síntomas de la neumonía por actinomicosis, que se encuentra en tratamiento con amoxicilina, pero tiene una respuesta farmacológica deficiente y un cuadro clínico de 3 días de evolución consistente con astenia, adinamia, dorsalgia, cefalea y un episodio de hemoptisis. Se inicia el tratamiento con dipirona sódica en una solución inyectable de $2,5 \mathrm{~g} / 5 \mathrm{~mL}$, acetaminofén en tabletas de $500 \mathrm{mg}$, cloruro de sodio al $0,9 \%$ en bolsa de $500 \mathrm{~mL}$, acetilcisteína de $300 \mathrm{mg} / 3 \mathrm{~mL}$ en una solución inyectable y ampicilina $1 \mathrm{~g} /$ sulbactam $0,5 \mathrm{~g}$ sódica en polvo para reconstituir. Debido a que se considera que el paciente presenta un cuadro nuevo de EPOC sobreinfectada exacerbada, se ordena una baciloscopia seriada, la cual es negativa, un gram y un cultivo que resultan también negativos; la flora orofaríngea resulta normal y la proteína C-reactiva es de 5,4 mg/L; también se realizan una radiografía de tórax (Figura 1C) y una TAC de tórax contrastada (Figura 2). Los hallazgos están relacionados con el diagnóstico de neumonía por actinomicosis. 

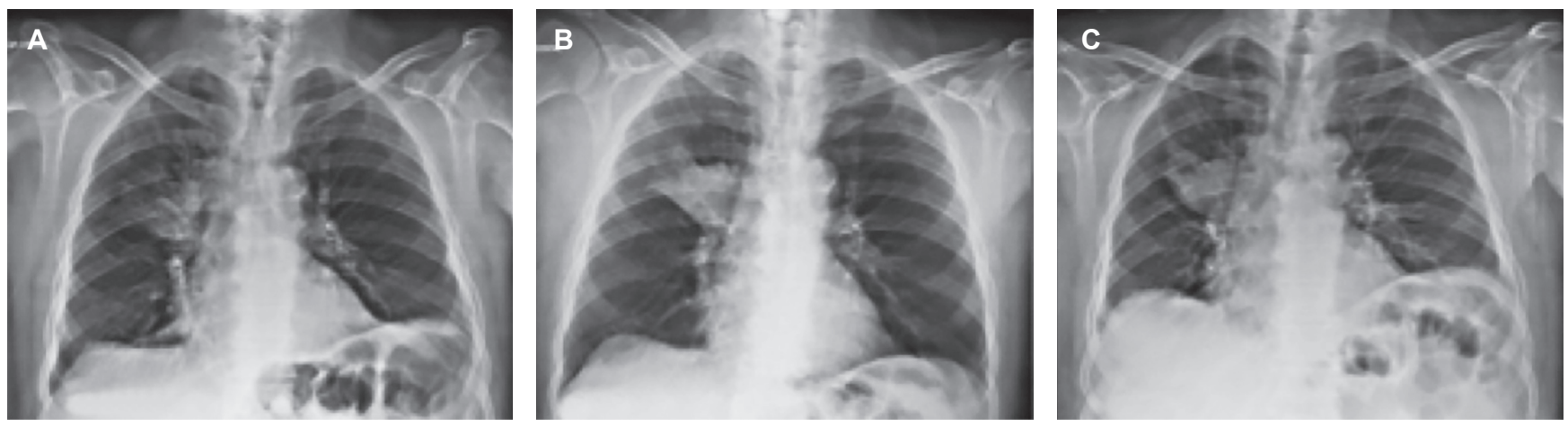

Figura 1. Radiografías de tórax. A. Consolidación inflamatoria pulmonar por la presencia de un broncograma aéreo, atelectasias subsegmentarias y una elevación del hemidiafragma izquierdo. B. Consolidación en el lóbulo superior derecho en el segmento anterior. C. Masa parahiliar derecha y opacidades reticulares en ambos lóbulos inferiores, con una elevación del hemidiafragma izquierdo.
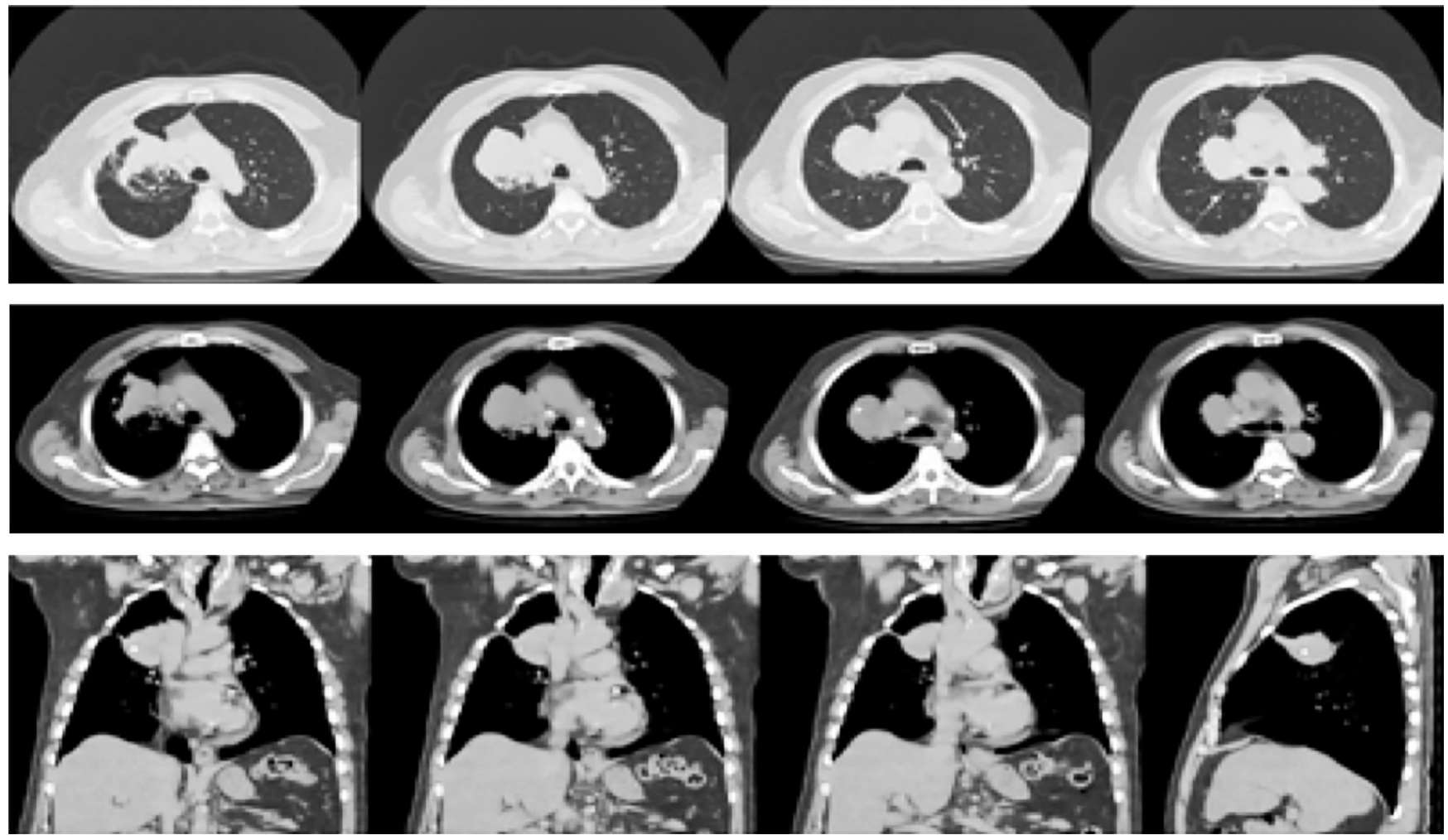

Figura 2. Tomografía de tórax. Masa con densidad del tejido blando de contornos lisos en el segmento apical del lóbulo superior derecho, que produce un compromiso circunferencial del bronquio segmentario, un engrosamiento de las paredes bronquiales con bronquiectasias en lóbulos inferiores y adenopatías hiliares bilaterales, algunas de ellas calcificadas.

Actualmente, el paciente lleva 4 meses en tratamiento farmacológico con amoxicilina para la actinomicosis pulmonar.

\section{Discusión}

Los factores de riesgo más comunes en los pacientes con actinomicosis pulmonar son la edad (entre 30-60 
años), sexo masculino, mala higiene oral, alteraciones pulmonares estructurales (enfisema, bronquiectasias), diabetes, inmunosupresión, uso de esteroides y bifosfonatos, leucemia con quimioterapia, VIH, trasplante de riñón y corazón, alcoholismo, daño tisular local causado por trauma, cirugía reciente y exposición a la radiación (1). En el caso presentado se conocen factores de riesgo como el sexo y la presencia de bronquiectasias, lo cual facilitó la presencia de la actinomicosis pulmonar.

El cuadro clínico que surge a partir de la aspiración de Actinomyces israelii desde la boca está influenciado por su patogenicidad y por los factores de riesgo anteriormente nombrados. La presentación clínica es variada; puede simular desde un cuadro infeccioso banal hasta una neoplasia, pero el cuadro clínico más común se presenta con tos productiva, fiebre, dolor en el pecho y pérdida de peso (9).

La afectación pulmonar, a pesar de ser poco frecuente, suele aparecer como un cuadro de condensación crónica que no mejora con el tratamiento antibiótico convencional, lo cual se evidencia en el cuadro clínico del paciente, quien, a pesar de contar con un tratamiento óptimo, presentó una respuesta pobre e incluso llegó a la reconsulta.

A menudo, la sintomatología se confunde con una neoplasia o una tuberculosis, y la mayoría de los estudios generalmente se centran en la búsqueda de estas patologías. Esto ocurre debido a que el diagnóstico de la actinomicosis es difícil de establecer, porque, en las primeras etapas de la infección, las imágenes muestran signos inespecíficos y no diagnósticos, como ocurrió con el paciente.

El diagnóstico se establece, en muchas ocasiones, después de haber agotado un importante número de pruebas complementarias, incluyendo la biopsia pulmonar, en la cual la presencia de gránulos de azufre es un indicador alto de actinomicosis (9-11).

El diagnóstico definitivo se realiza por medio de un estudio microscópico de secreciones de la lesión, donde la tinción especial que confirma el diagnóstico de actinomicosis es la presencia del fenómeno de Splendore-Hoeppli. Este fenómeno es patognomónico de actinomicosis y consiste en una imagen de interfase entre colonias bacterianas y el exudado de polimorfonucleares, con un depósito de material eosinófilo (12).

En la radiografía se han descrito diversos cambios que varían desde infiltrados alveolares hasta grandes masas, los cuales son similares a los hallazgos encontrados en el paciente, así como las imágenes de TAC de tórax con presencia de infiltrados y masas $(11,12)$.

En cuanto al tratamiento, no existe un consenso sobre la duración de la terapia. Es una enfermedad tratable y un diagnóstico correcto y temprano permite una buena respuesta al tratamiento antibiótico. El tratamiento puede dividirse en farmacológico y quirúrgico, dependiendo de la variedad clínica de la enfermedad.

El tratamiento farmacológico generalmente se basa en el uso de betalactámicos del tipo penicilínicos, ya sea en forma parenteral $u$ oral, como la amoxicilina, la penicilina $\mathrm{G}$ y la ampicilina. En los pacientes alérgicos a las penicilinas se pueden usar macrólidos o lincosamidas; en el caso del paciente que no sea alérgico, el tratamiento consiste en la administración de amoxicilina por vía oral durante un período de 6 meses.

Por otro lado, el tratamiento quirúrgico solamente está indicado en el caso de nódulos o lesiones tumorales; sin embargo, el tratamiento clásico suele ser el farmacológico, el cual incluye inicialmente la administración de un antibiótico por vía intravenosa durante 2-6 semanas, seguida de la administración de un antibiótico por vía oral para completar 4-6 meses $(10,12)$.

\section{Conclusiones}

A pesar de la baja frecuencia y sospecha de la actinomicosis pulmonar en los pacientes que presentan cuadros clínicos compatibles con neumonía, bronconeumonía o cuadros atípicos que no orientan a un diagnóstico definitivo, con una afectación pleuropulmonar de curso subagudo y con baja respuesta al tratamiento asignado, es aconsejable realizar cultivos de muestras en medios anaerobios e informar al microbiólogo asignado la sospecha de una infección por actinomicosis. De la misma forma, es fundamental el uso de paraclínicos que incluyan la toma de una radiografía de tórax, una PET/CT y una biopsia guiada por tomografía, con 
el fin de brindar un diagnóstico oportuno y un tratamiento adecuado, temprano e individualizado que mejore la respuesta y el pronóstico del paciente, con lo cual se evita la reconsulta y la exposición a infecciones adicionales. En cuanto al tratamiento actual, no existe un estándar definido aún, pero se recomienda el uso de betalactámicos del tipo penicilina, ya sea en forma parenteral u oral como amoxicilina, penicilina $\mathrm{G}$ oral y ampicilina.

\section{Conflicto de intereses}

No existen conflictos de intereses.

\section{Referencias}

1. Wong VK, Turmezei TD, Weston VC. Actinomycosis. BMJ 2011;343:d6099. doi:10.1136/bmj.d6099

2. Arenas R. Actinomicosis. En: Micología Médica Ilustrada. 5.a ed. México D. F: McGraw-Hill; 2014. p. 303-305.

3. Bouillouda F, Le Clechb G. Actinomicosis ceervicofacial. EMC-Otorrinolaringología. 2010;39(4): 1-5. doi: 10.1016/ S1632-3475(10)57838-X

4. Mabeza GF, Macfarlane J. Pulmonary actinomycosis. Eur Respir J. 2003;21:545-551. doi: 10.1183/09031936.03.00089103.
5. Dalhoff K, Wallner S, Finck C, Gatermann S, Wiessmann KJ. Endobronchial actinomycosis. Eur Respir J. 1994;7:1189-91. doi: 10.1183/09031936.94.07061189.

6. Llombart M, Chiner E, Signes-Costa J, Arriero JM, GómezMerino E, Andreu A, et al. Actinomicosis torácica: una vieja entidad con nuevas expresiones clínicas. An. Med. Interna. 2005;22(3):28-33.

7. Brown JR. Human actinomycosis: a study of 181 subjects. Human Pathol. 1973;4:319-330. doi:10.1016/s00468177(73)80097-8.

8. Drake DP, Holt RJ. Childhood actinomycosis. Report of 3 recent cases. Arch Dis Child. 1976 Dec;51(12):979-81. doi: 10.1136/adc.51.12.979.

9. Gómez M, Ray C, Allenspach L, Kvale P. Endobronchial Actinomycosis Associated With a Foreign Body. Journal of Bronchology. 2005;12(1):54-55. doi: 10.1097/01.laboratory.0000149182.39322.71.

10. Bouza Y, Jam BC, Tartabull Y. Actinomicosis pulmonar. Presentación de un caso. Medisur. 2015;13(6):795-9.

11. Ramírez F, Barrera EC, Méndez RY. Actinomicosis pulmonar, Una enfermedad olvidada. Acta Med Colomb. 2010;35(3):132-134.

12. Flores A, Parrilla I, Urdes E, Quintana V, Gutiérrez V. et al. Prevención, Diagnóstico y Tratamiento de la Actinomicosis en el adulto. México D. F.: IMSS, CENETEC; 2010:7. Disponible en: http://www.cenetec.salud.gob.mx/descargas/gpc/ CatalogoMaestro/480_GPC_Actinomicosis/GER_ACTINOMICOSIS.pdf 